Japantse Prichologzal Research

1983, Vol. $25, \mathrm{No}, 2,59-68$

\title{
Role of form information in stroboscopic motion perception studied by masking the figural processes
}

\author{
HIDEKO OHMURA and KATSUO SAIGO \\ Department of Psychology, Kyushu Institute of Design, Minami-ku, Fukuoka 815
}

\begin{abstract}
The role of form information in stroboscopic motion perception was studied with different pairs of geometrical transforms of the stimuli to be successively presented, of which either one or both were suppressed by overlapping masking discs as in the metacontrast paradigm. When form information was reduced to either one of the stimuli, different types of stroboscopic motion were visible depending on the types of geometrical transformation where, however, the form information of the suppressed figure tended to conform to that of the unsuppressed one, and consequently the simple translation increased while rotation, two-dimensional or three-dimensional, decreased as compared with that under no-masking conditions. Which one of the stimuli, the first or the second, was suppressed was indifferent to the generation of motion. If two stimuli were both suppressed, no stroboscopic motion was observed.
\end{abstract}

Key words: stroboscopic motion, type of gcometric transformation, form information, translation, three-dimensional rotation, two-dimensional rotation, plastic deformation.

In a few studies (Neuhaus, 1930 Orlansky, 1940; Squires, 1959; Metzger, 1954), using disparate shapes as stimuli in the situation of stroboscopic motion, it has generally been found that the probability of seeing motion lessened. Kolers and Pomerantz (1971) dealt with the types of perceived motion (translation, plastic deformation of contours and rotation of rigid shapes in depth) between two disparate stimuli, but they did not decide how each type of perceived motion corresponds to the type of geometric transformation. According to them, the figural properties of stimuli do not affect the probability of seeing motion. Increasing stimulus duration increases the probability of all three, but changes in depth occur at longer interstimulus intervals. Thus they regarded the temporal factor as primary in stroboscopic motion and the figural properties of stimuli as secondary.

The authors (Ohmura, 1981; Ohmura \& Saigo, 1982) investigated what visual information specifies the types of pcrceived motion and how they associate with the temporal condition (duration and $\mathrm{SOA}$ ). In these studies, using six different kinds of stimulus pairs, the probability of the types of perceived motion between them was obtaincd under various temporal conditions. The first stimulus was always a square in each pair and the second stimuli were geometrically transformed figures of the square: In the stimulus pair (a); the same square as the first stimulus by identily transformation, (b); a trapezoid by projective transformation, (c); a scalene hexagon by projective transformation of a cube, (d); a rectangle by affine transformation, (e); a triangle by topological transformation, and (f); a diamond by congruent transformation. The results were as follows. (1) Four kinds of seeing motion were found; i.e., translation, plastic deformation, rotations in three-dimensional space and on two-dimensional plane. (2) Some of these types of motion were $u$ niquely corresponded to a specific geometric transformation, but some were not. Translation corresponded to identity transformation, while plastic deformation corresponded to affine or to topological transformation. Plastic deformation and rotations also corresponded to projective or to congruent transformation. (3) There was no effect of stimulus durations in the case of unique correspondence. In other cases, 
rotations, either two- or three-dimensional, were relatively superior to plastic deformation as the duration increased. The authors concluded that there is the correlation between the type of geometric transformation of stimuli and those of stroboscopic motion therefrom.

These findings suggest that form information of both stimuli is important to decide the type of perceived motion. Von Grünau (1978a) has already indicated that when form information of one of two stimuli is suppressed by metacontrast masking, without interfering with the motion information, apparent motion does still occur quite easily, and that, in comparison with the condition of nonmasking, the probability of seeing motion decreases when the first stimulus is masked, but it increases when the second is masked. Moreover, von Grünau (1979) came to the conclusion that optimal stroboscopic motion is no longer possible when form information of both stimuli is suppressed. Thus, he (1978a, 1979) also had already shown the importance of form information of stimuli in stroboscopic motion.

However, since in his experiments both stimuli consisted of identical pairs consisting of disks, the type of perceived motion was limited only to the translation. Therefore, it was not possible to analyse qualitatively how the form information of each of the stimuli was actually contributing to the type of perceived motion.

In the present experiments, an attempt was made to assess the contribution of form information of each stimulus to the type of perceived motion by measuring the probability of seeing motion "in general "-regardless of the types of perceived motion-and that of the type of perceived motion, when one of the two disparate stimuli in shape or both of them were also masked by metacontrast.

To accomplish this aim, two experiments were conducted. In Exp. I were measured the probabilities of realizing the different types of stroboscopic motion be- tween two disparate shapes under nonmasking (the control experiment), and in Exp. II were measured those under metacontrast masking.

\section{Experiment I}

The design of this experiment closely followed ours in earlier study (Ohmura \& Saigo, 1982), but temporal parameter, particularly, stimulus duration was different. In our earlier experiment, another stimulus durations werc used, but these were not available to timing necessary to cause optimal masking in Exp. II. In order to compare directly the results under nonmasking condition (Exp. I) with those under masking conditions (Exp. II), data with new stimulus duration, which gave optimal masking, were required.

\section{Method}

Apparalus. Using a four-channel tachistoscope, the first stimulus figure (visual angle $1^{\circ} \times 1^{\circ}$, luminance $3 \mathrm{~cd} / \mathrm{m}^{2}$ ) on the left side on the plane of stimulus presentation and the second one (the same visual angle and luminance as the first) on the right side were successively presented under specified temporal conditions. The spatial extent between each center of both stimuli subtended visual angle of $3^{\circ}$. On the basis of the findings that the inhibitory effect of the transient channel (the processing of motion) on the sustained channel (the processing of form) is larger in the periphery than in the fovea (von Grunau, $1978 b$ ), a small fixation point, FP (luminescent diode, diameter $0.1^{\circ}$, luminance $6 \mathrm{~cd} / \mathrm{m}^{2}$ ) was set on the left, visual angle $2^{\circ}$ from the center of the first stimulus. The stimuli were all plain figures, which were obtained by covering a luminescent panel with the black paper cut out to generate the proper stimulus patterns. Observation was with the left eye. The distance from subjects' eye to the stimulus surface was $1 \mathrm{~m}$.

Temporal conditions. The durations of the first stimulus and the second were 
fixed to $80 \mathrm{~ms}$ at all time. This value was chosen in relation to the temporal conditions for the optimal masking in Experiment II (refer to Method of Experiment II). The time intervals between the onsets of two stimuli (SOA) were 40, 80, 120, $160,200,240,280$ and $320 \mathrm{~ms}$. The time interval from the offset of the second stimulus to the following onset of the first stimulus (intercycle interval: ICI) was set at $800 \mathrm{~ms}$.

Subjects. Sevin college students served as subjects. All had normal vision, and were well acquainted with the experiment of apparent motion.

Stimulus figure. As shown in Fig. 1, three kinds of stimulus pairs (a), (b) and (c) were used. The first stimuli on the left were all squares of the same size, but the shapes of the second stimuli were geometrically transformed figures of the first differently. In the stimulus pair (a), the second stimulus was exactly the same square obtained by identity transformation. In the stimulus pair (b), it was a trapezoid obtained by projective transformation, in which the square was rotated $30^{\circ}$ about $Y$-axis three-dimensionally (in depth) and then projected on the picture plane. In the stimulus pair (c), it was a diamond obtained by congruent transformation in which the square was rotated $45^{\circ}$ clockwise from the vertical axis on the twodimensional plane.

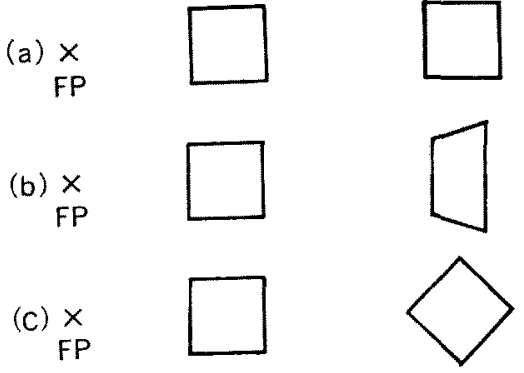

Fig. 1. Three ways of geometric transformation in stimulus pairs used in Exp. I. (a): identity transformation, (b): projective transformation, (c): congruent transformation.
Procedure. Two stimuli were presented in the dark room under the stimulus duration of $80 \mathrm{~ms}$, eight steps of SOA and ICI of $800 \mathrm{~ms}$. Preceding the experiment, each subject was darkadapted for $10 \mathrm{~min}$. Then, according to the given instruction, he was required to report whether the smooth motion of a single object from the position of the first stimulus to that of the second was seen, and what type of stroboscopic motion was.

Twenty-four conditions, defined by the combinations of the eight steps of SOA with three kinds of stimulus pair, were presented five times in random order. A total 120 trials, therefore, were given to each subject. The stimuli were repeatedly given until subjects were able to respond whether motion was seen or not. Then they were also asked to report the type of perceived motion whenever stroboscopic motion occurred between both stimuli.

The proportion of the number of perceived motion to the total trials under each condition was calculated. The results were analyzed using analysis of variance procedure.

\section{Results}

In Fig. 2, the probability of seeing motion "in general" for each of three different stimulus pairs is shown as a function of eight different SOAs.

As clarified in Fig. 2, the obtained curves are similar in shape and all of them show the inversed $U$-shaped functions

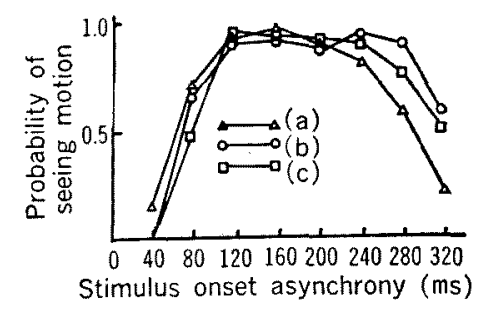

Fig. 2. The probability of seeing motion " in general " obtained for three kinds of stimulus pair. (a): identity transformation, (b): projective transformation, (c): congruent transformation. 
$\left(\chi^{2}(2)=1.54, \quad p>0.10\right)$. This fact indicates that the probability of seeing motion "in general" is independent of the types of geometric transformation in stimuli. These results also agree with the earlier experimental findings in which the form properties of figures were regarded as a secondary factor in stroboscopic motion (Kolers \& Pomerantz, 1971; Kolers, 1972; Ohmura \& Saigo, 1982).

The probability of seeing each type of motion for the stimulus pair (b) (projective transformation) is shown in Fig. 3-(b), and that for the stimulus pair (c) (congruent transformation) in Fig. 3-(c). For the stimulus pair (a) (identity transformation), only the traditional $\beta$ motion, namely the translation of a single square from the position of the first stimulus to that of the second, was seen, because the shapes of both stimuli were identical. In Fig. 3, therefore, were not plotted the results for the stimulus pair (a).

As shown in Fig. 3-(b), in the case of stimulus pair (b), two types of motion were caused; the plastic deformation and the three-dimensional rotation. And there was a difference of more than 120 ms between the two values of SOA, each of which corresponded to the peak in probability of each type of perceived motion.

In the case of stimulus pair (c), two types of motion, the plastic deformation and the rotation in the picture plane, were caused.

\section{Experiment II}

The purpose of this experiment was to examine the following questions.

(1) What happens to the type of perceived motion when one of disparate shapes is suppressed by metacontrast masking?

(2) What happens to the one when both are masked?

The results of Exp. II under the masking conditions were compared with these of Exp. I under the nonmasking condition.

\section{Method}

Apparatus. As shown in Fig. 4, the first stimulus $\left(\mathrm{S}_{1}\right.$ : visual angle $1^{\circ} \times 1^{\circ}$, luminance $\left.3 \mathrm{~cd} / \mathrm{m}^{2}\right)$, the second stimulus $\left(\mathrm{S}_{2}\right.$ : the same visual angle and luminance as $S_{1}$ ) and the masking stimuli (the first masking stimulus $M_{1}$, the second masking stimulus $\mathrm{M}_{2}$ : both of them visual angle $1.8^{\circ}$, luminance $3 \mathrm{~cd} / \mathrm{m}^{2}$ ) to suppress one or both of the stimuli were presented successively under several temporal conditions. $\mathrm{S}_{1}-\mathrm{M}_{1}$ and $S_{2}-M_{2}$ were respectively arranged concentrically. The other details were the same as those in Exp. I.

Temporal conditions. The durations were as follows: $S_{1}, S_{2}=80 \mathrm{~ms} . M_{1}, M_{2}=$ $100 \mathrm{~ms}$. These values were determined in pilot studies with the aim of avoiding unwanted motion between $S$ and $M$, which otherwise would interfered with the motion between $S_{1}$ and $S_{2} . S_{1}-M_{1}$ and $S_{1}-M_{2}$ intervals (SOA for optimal masking) were different among $\mathrm{Ss}$, so these were individually determined. SOAs $\left(\mathrm{S}_{1}-\mathrm{S}_{2}\right.$ motion) and ICI were the same as those in Exp. I.

Subjects. The same seven students participating in Exp. I served as subjects.

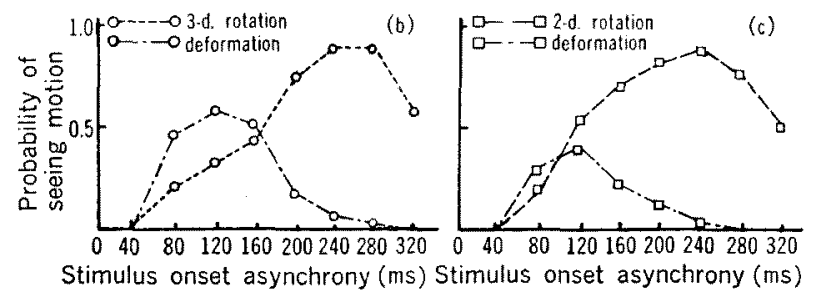

Fig. 3. The probability of types of perceived motion obtained for the stimulus pairs (b) and (c). 
A

masking of the first stimulus

(a) $\underset{F P}{\mathrm{SP}} \mathrm{M}$

(b) $\times$ FP
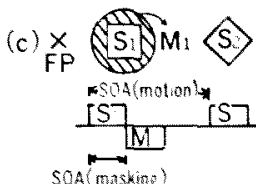

$B$

masking of the second stimulus

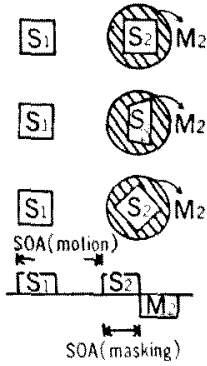

Fig. 4. Spatial and temporal stimulus arrangement used in Exp. II. Arrangement $A$ : with masking of the first stimulus $\left(S_{1}\right)$ by masking stimulus $\left(\mathrm{M}_{1}\right)$, Arrangement $\mathrm{B}$ : with masking of the second stimulus $\left(S_{2}\right)$ by masking stimulus $\left(M_{2}\right)$. Arrangement with masking of both $S_{1}$ and $S_{2}$ is omitted here.

Stimulus figures. As shown in Fig. 4, added to the three kinds of stimulus pair in Exp. I, three kinds of masking stimulus pair belonging to each stimulus pair were prepared. Every masking stimulus was made by covering a lumincscent panel with the black paper cut out in the figures of the masking stimulus.

Procedure. At first, it was determined for each subject which temporal interval (SOA masking) between a stroboscopic motion stimulus and a masking stimulus gave optimal masking. This measurement was made as follows; SOA between a stimulus $\left(\mathrm{S}_{1}\right.$ or $\left.\mathrm{S}_{2}\right)$ and a masking stimulus $\left(M_{1}\right.$ or $M_{2}$ ) was changed gradually from $40 \mathrm{~ms}$ to $180 \mathrm{~ms}$ in $20 \mathrm{~ms}$ steps. At each of SOA, subject was required to report
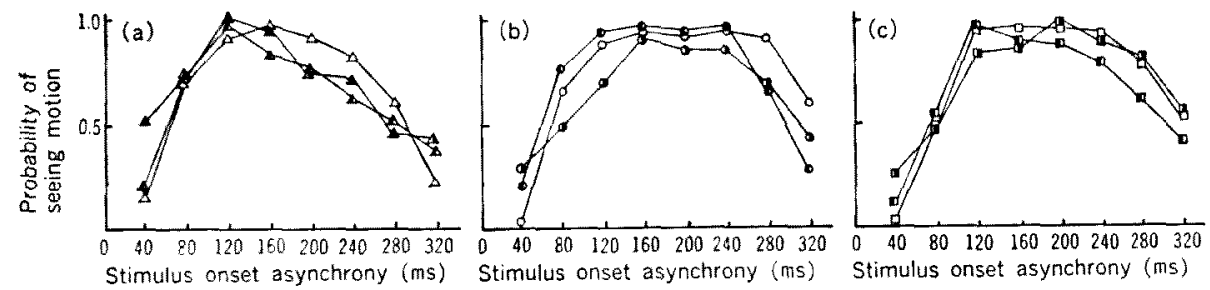

Fig. 5. The probability of seeing motion "in general" obtained for the stimulus pairs (a), (b) and (c) under the conditions without masking $(\triangle, O, \square)$, with masking of the first stimulus $(\mathbf{C}, \mathbf{D}, \mathbf{D})$

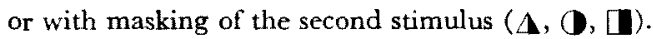
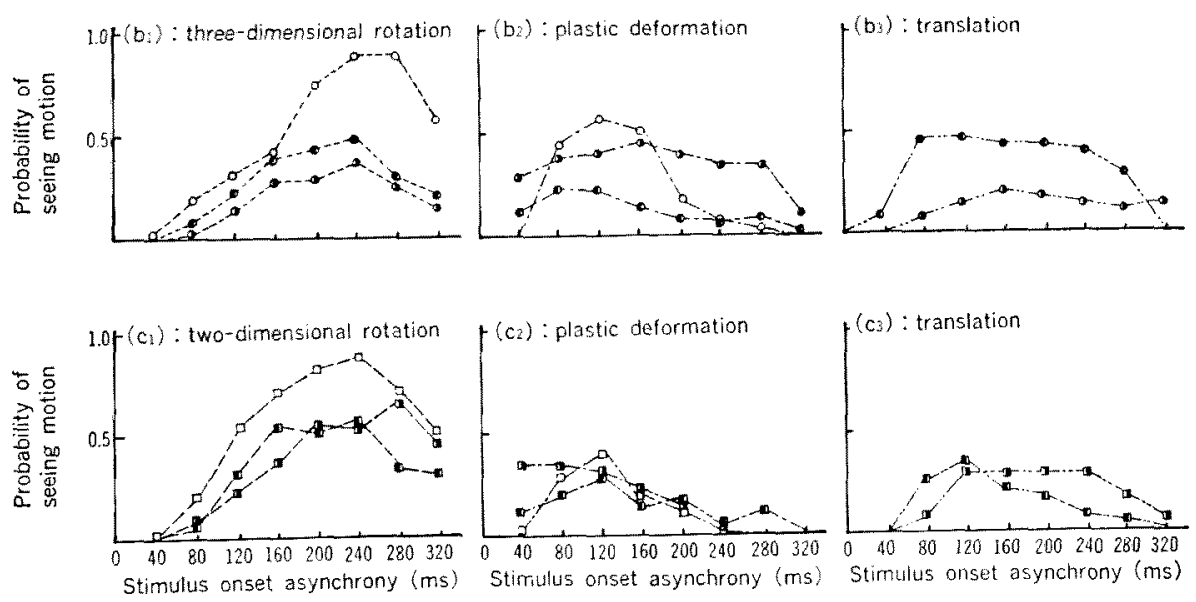

Fig 6. The probability of types of perceived motion obtained for the stimulus pairs (b) and (c) under the conditions without masking $(O, \square)$, with masking of the first stimulus $(0, \square)$ or with masking of the second stimulus $(\mathbf{O}, \mathbf{\square})$. 
"yes" or " no", to the question: "Do you see the stimulus $\left(\mathrm{S}_{1}\right.$ or $\left.\mathrm{S}_{2}\right)$ ?" Five trials for each of the cight steps of SOA, as above mentioned, that is, 40 judgments in total, required randomly for each subject. The value of SOA corresponding to the answers "no" was decided to be SOA of the optimal masking for each subject. All the values of SOA were within the range from $60 \mathrm{~ms}$ to $100 \mathrm{~ms}$. Therefore, the scatter of the range was not so large as to exceed the range (40 $\mathrm{ms}-$ $300 \mathrm{~ms}$ ) of the values of SOA between $S_{1}$ and $S_{2}$ as the independent variable common to each subject, and its changing step (40 ms). (Here, we omit showing the values of the optimal masking SOA for each subject.) These determined values stayed constant when SOA (motion) was varied.

Then, the probability of seeing motion "in general " and of the types of perceived motion between $S_{1}$ and $S_{2}$ were measured, with masking of $S_{1}$, with masking of $S_{2}$ and with masking of both $S_{1}$ and $S_{2}$. Three kinds of stimulus pair and three masking conditions were combined to total nine sessions. The order of the nine sessions was counterbalanced among subjects. In every session, five times for each of the eight steps of SOA, forty trials in total, were given for each subject.

The other procedure was the same as that in Exp. I.

\section{Results}

When both the first and the second stimulus were suppressed by metacontrast masking, stroboscopic motion did not occur for any stimulus pair.

In Fig. 5, the probability of seeing motion "in general" under each condition, with nonmasking, with the first stimulus masking and with the second stimulus masking, was plotted as a function of eight SOAs, for each of the stimulus pairs (a), (b) and (c). (As for the results with the nonmasking, those in Exp. I were quoted.) Comparing the results under the two test conditions of the masking with those under the condition of the nonmasking, the followings were found: For all the stimulus pairs (a), (b) and (c), as shown in Fig. 5-(a), -(b) and $-(c)$, the differences among threc conditions were not significant ((a): $\chi^{2}$ (2) $=2.38, p>0.10 ;(\mathrm{b}): \chi^{2}(2)=5.36, p>$ $0.05 ;(\mathrm{c}): \chi^{2}(2)=0.28, p>0.10$ respectively). Thus, for the probability of secing motion "in general", there was no difference between when one of the two stimuli was masked and when both stimuli were not masked.

In Fig. 6, the probability of each type of perceived motion was plotted as a function of eight SOAs, under each condition with the nonmasking and the masking, for the stimulus pairs (b) and (c).

As for the stimulus pair (a), since two stimuli were identical in shape, the type of perceived motion was always limited to the translation only. Therefore, these results were the same as those in Fig. 5-(a), and were omitted here.

In Fig. 6- $\left(b_{1}\right),-\left(b_{2}\right)$ and $-\left(b_{3}\right)$ were shown the results for the stimulus pair (b). As indicated in these Figs., the types of perceived motion were different both in the number and in the probability, between with the masking conditions and with the nonmasking condition. That is, under the nonmasking condition, only two types of motion occurred; the threedimensional rotation and the plastic deformation. On the other hand, under the masking conditions, the translation was also observed besides the above two types of it, and there was a remarkable difference also in the form of the curves concerning these types of motion. For example, the probability of three-dimensional rotation decreases significantly with the masking more than with the nonmaskings $\left(\chi^{2}(2)=9.0, p<0.01\right.$; between the nonmasking and the first stimulus masking: $C R=2.26, p<0.05$; between the nonmasking and the second stimulus masking: $C R=1.65, p<0.05$ ). However, 
as for the degree of decreasing, there was no significant difference between the condition of the first stimulus masking and that of the second stimulus masking ( $C R=$ $0.50, p>0.30)$.

In Fig $6-\left(c_{1}\right),-\left(c_{2}\right)$ and $-\left(c_{3}\right)$, the probability of the types of perceived motion for the stimulus pair (c) was shown. As shown in these Figs., like the stimulus pair (b), the types of perceived motion were also different in the number and in the probability between with the masking and with the nonmasking. That is, the two types of motion occurred with the nonmasking; the two-dimensional rotation and the plastic deformation. On the other hand, in addition to the above two types of it, the translation also occurred with the masking conditions. Moreover, the probability of the two-dimensional rotation decreased more significantly with the masking conditions than with the nonmasking ( $\chi^{2}(2)=6.85, p<0.05$; between the nonmasking and the first stimulus masking: $C R=2.26, p<0.05$; between the nonmasking and the second stimulus masking: $C R=2.04, p<0.05$ ). However, as for the degree of decreasing, there was no significant difference between the condition of the first stimulus masking and that of the second stimulus masking ( $C R=$ $0.41, p>0.30)$.

\section{Discussion}

In Exp. I, as the control experiment, the probability of seeing motion "in general" and that of the types of perceived motion were measured without masking, under the same temporal and spatial conditions as those in Exp. II. It was indicated that the probability of seeing motion "in general" was not related to the types of geometric transformation in stimuli, and that between types of geometric transformation and those of stroboscopic motion there was a certain correspondence; identical transformation to translation, projective transformation to three-dimensional rotation and plastic deformation, or congruent transformation to two-dimensional rotation and plastic deformation. These results confirmed those in our earlier studies (Ohmura \& Saigo, 1982), in which similar six kinds of stimulus pairs by geometric transformation were used.

The purpose of Exp. II was to cxamine how these confirmed results change in the situation of metacontrast masking. Many psychologists, mainly from the Gestalt school, regarded the perception of form to be a necessary prerequisite (Van der Waals \& Roelofs, 1930; von Schiller, 1933), and it has since been demonstrated that stroboscopic motion does not depend on the prior conscious perception of the form of the inducing stimuli (Kolers, 1963, 1972; Anstis, 1970). The contribution, however, of form information from both stimuli to the types of perceived motion was evidenced by the fact that the shape of the object changes smoothly from the shape of the first to that of the second stimulus, when the two stimuli have different shapes (Kolers \& von Grünau, 1976; Ohmura, 1981; Ohmura \& Saigo, 1982).

Von Grünau (1978a) has shown the following: When form information of one of the two inducing stimuli was suppressed by metacontrast masking, without interfering with the motion information, motion did still occur quite readily, and that, in this case, the likelihood of seeing stroboscopic motion became less likely when the first stimulus was masked in the metacontrast situation and more likely when the second stimulus was masked. But masking of both stimuli abolished the perception of motion. From these results, von Grünau concluded that: Though the form information of the first stimulus was suppressed (the disk was not seen), some information that could be used by the motion-generating system was not suppressed (motion or position information). The fact that the likelihood of seeing mo- 
tion decreased, when the first stimulus was masked, indicates that the form information of the first stimulus is important for the generation of stroboscopic motion. The fact that the likelihood of seeing motion increased, when the second stimulus was masked, indicates that this form information usually interferes with the generation of motion.

The present study investigated the case in which form information of one of two stimuli or both of them is suppressed by metacontrast masking to testify the importance of form information for the perception of motion. It was shown that stroboscopic motion between two stimuli does occur quite readily, even when one of both stimuli is masked, but it does not occur when both stimuli are masked. These results agree with those of von Grunau (1978a), but it was different that the probability of seeing motion "in general " was obtained with the masking of one of two stimuli as well as without the masking. From this, it is clear that the involvement of form information of the first stimulus in motion information is equivalent to that of the second stimulus.

Moreover, it was found that the correspondence between the types of geometric transformation in stimuli and those of perceived motion without the masking of both stimuli was not always retained with the masking of one of them. For the disparate stimulus pairs (projective and congruent transformation), the translation, in addition to the rotation and the plastic deformation, was still more apparent with the masking. For the identical stimulus pair (identity transformation), however, the correspondence was retained, with the masking, in which only the translation still appeared. In these cases, the probability of the types of two- or threedimensional rotation decreased, while that of the translation was increased. This tendency appeared irrelevant to whether the first or the second stimulus is suppressed by metacontrast masking. When form information of both stimuli is suppressed, optimal stroboscopic motion was no longer possible.

Thus, even when form information of one of two stimuli was suppressed by metacontrast masking, the different types of perceived motion (the shape of the object changes smoothly from the shape of the first stimulus to that of the second) did still occur - though the probability of translation increases with decreasing that of rotation and plastic deformation-. This fact shows that the suppressed form information can still participate in stroboscopic motion.

Therefore, considering that motion did not occur by the masking of both stimuli, it is suggested that the perception of at least one of both stimuli is necessary for the occurrence of stroboscopic motion, and that the participation of the suppressed form information of one stimulus is coordinated with the perception of the form of the other stimulus. The masking of one of both stimuli clearly brought about changes in both the types of perceived motion and the probability of them. Concerning these changes, the involvement of form information of the first stimulus in motion information is equivalent to that of the second stimulus. This also disagrces with von Grünau's findings (1978a) that form information of the first stimulus is important for the generation of stroboscopic motion and that of the second interferes with the generation of motion.

\section{Summary}

Two experiments were conducted to examine how form information of each stimulus (in types of geometric transformation) actually contributes to the probability of seeing motion " in general" and the type of perceived motion. In Exp. I, the probability of seeing motion " in general" and that of the types of perceived motion was measured under the nonmasking condition, and in Exp. II, 
they were measured under the metacontrast masking. These results were as follows:

(1) When the form information of one of two stimuli was suppressed by metacontrast, stroboscopic motion does occur quite easily. However, when the form informations of both stimuli were suppressed, stroboscopic motion was no longer possible for any stimulus pair.

(2) As for the probability of sceing motion "in general", there was no difference between the condition without masking and those conditions in which the first or the second stimulus was masked. Therefore, it was independent of the type of geometric transformation of stimuli.

(3) When one of two stimuli was masked, the type of perceived motion was still actually caused according to the type of geometric transformation of stimuli.

(4) For the stimulus pair in identity transformation, the type of perceived motion was always limited to translation only, whether the form information of stimuli was suppressed or not. For the stimulus pair in projective transformation, the translation, in addition to both the threedimensional rotation and the plastic deformation under the nonmasking condition, occurred under the masking conditions. With the masking, the probability of threedimensional rotation decreased for the occurrence of the translation. For the stimulus pair in congruent transformation, the translation, in addition to both the two-dimensional rotation and the plastic deformation under the nonmasking condition, also occurred under the masking conditions. With the masking, the probability of two-dimensional rotation decreased for the occurrence of the translation.

In short, it was suggested that there was no difference between the involvement of the form information of the first stimulus and that of the second in the generation of motion, that, with the masking of one of stimuli, stroboscopic motion might depend on the coordination of the suppressed form information of one stimulus with the nosuppressed form information of the other stimulus, and that, with the masking of both stimuli, the coordination of both the suppressed form information can not be there.

\section{References}

Anstis, S. M. 1970 Phi movement as a substraction process. Vision Research, 10, 1411-1430.

Grünau, M.W. von 1978a Dissociation and interaction of form and motion information in the human visual system. Vision Research, 18, 1485 1489.

Grünau, M.W. von $1978 \mathrm{~b}$ Interaction between sustained and transient channels: Form inhibits motion in the human visual system. Vision Research, 18, 197-201.

Grünau, M. W. von 1979 Form information is necessary for the perception of motion. Vision Research, 19, 839-841.

Kolers, P. A. 1963 Some differences between real and apparent visual movement. Vision $R e$ search, 3, 191-206.

Kolers, P. A. 1972 Aspects of motion perception. New York: Pergamon Press.

Kolers, P. A., \& Grünau, M. W. von 1976 Shape and color in apparent motion. Vision Research, 16, 329-335.

Kolers, P. A., \& Pomerantz, J. R. 1971 Figural change in apparent motion. Journal of Experimental Psychology, 87, 99-108.

Metzger, W. 1954 Psychologie. 2 Aufl. Darmstadt: Dietrich Steinkopff.

Neuhaus, W. 1930 Experimentelle Untersuchung der Scheinbewegung. Archiv für die gesamte Psychologie, 75, $315-458$.

Ohmura, H. 1981 Effects of subjective contours in stroboscopic motion. Japanese Journal of $P_{s y}$ chology, 52, 223-239. (In Japanese with English abstract)

Ohmura, H., \& Saigo, K. 1982 The types of stroboscopic motion as determined by geometric transformations and temporal factors in stimulus presentation. Japanese Psychological Research, 24, $68-77$.

Orlansky, J. 1940 The effect of similarity and difference in form on apparent visual movement. Archives of Psychology, 85, 246.

Schiller, P. von 1933 Stroboskopische Alterna- 
tivversuch. Psychologische Forschung, 17, 179-214. Squires, P. C. 1959 Topological aspects of apparent visual motion. Psychologische Forschung, 26, 1-12.

Van der Waals, H. G., \& Rociofs, G. O. 1930
Optische Scheinbewegung. Zeilschrift für Psychologie und Physiologie der Sinnesorgane, 114, 241288.

(Received April 7, 1982; accepted May 14, 1983) 